\title{
Laparoscopy-assisted esophagoenteral anastomosis using endoscopic purse-string suture instrument "Endo-PSI (II)" and circular stapler
}

\author{
Shinsuke Usui, Kentaro Nagai, Susumu Hiranuma, Noriaki Takiguchi, Akio Matsumoto, \\ and Katsuhiro SAnADA \\ Department of Surgery, Tsuchiura Kyodo General Hospital, 11-7 Manabe-shinmachi, Tsuchiura, Ibaraki 300-0053, Japan
}

\begin{abstract}
It is said that laparoscopic esophagoenteral anastomosis is not easy. In particular, purse-string suture of the abdominal esophagus is difficult when using a circular stapler. We have developed an endoscopic purse-string suture instrument, the "Endo-PSI (II)", and the instrument was employed clinically during laparoscopy-assisted total gastrectomy. The device was inserted into the abdominal cavity through a 4-cm minilaparotomy of the epigastrium, and pneumoperitoneum was established by closing a Lap Disc. The Endo-PSI (II) was attached to the abdominal esophagus and a straight needle with a 2-0 polypropylene suture was passed through the device laparoscopically. After a purse-string suture of the abdominal esophagus was made, the abdominal esophagus was transected laparoscopically and the removed stomach was pulled out through the minilaparotomy. The anvil head of a circular stapler was inserted into the abdominal cavity through the minilaparotomy, and insertion of the anvil into the esophagus and ligation of the purse-string suture were performed laparoscopically, too. The combination of using a circular stapler for esophagojejunostomy and closure of the jejunal stump was also performed laparoscopically. Between May 2007 and May 2008, these products were used in 23 patients during laparoscopy-assisted total gastrectomy. There were no cases that required conversion to a conventional open procedure. The newly developed Endo-PSI (II) was useful for laparoscopic purse-string suture of the esophagus.
\end{abstract}

Key words Esophago-enterostomy - Esophagojejunostomy · Laparoscopy-assisted total gastrectomy - Gastric cancer · Purse-string instrument

\section{Introduction}

Up to now, there are several reports about laparoscopic esophagoenteral anastomoses. In many cases, side-to-

Offprint requests to: $\mathrm{S}$. Usui

Received: May 19, 2008 / Accepted: August 23, 2008 side anastomosis using an endoscopic linear stapler has been reported $[1,2]$. On the other hand, it is common to use a purse-string suture instrument (PSI) and circular stapler for esophagoenteral anastomosis in conventional open surgery. Why is a linear stapler preferable to a circular stapler in laparoscopic surgery? Mainly because attachment of the anvil head of a circular stapler to the abdominal esophagus is thought to be difficult in laparoscopic surgery. Especially, purse-string suture of the abdominal esophagus is said to be difficult, even if the procedure is performed manually. To overcome these difficulties, we developed an endoscopic PSI, the "Endo-PSI", in 2007 [3]. This device was designed for hand-assisted laparoscopic surgery (HALS). In the present study, we report the use of the "Endo-PSI (II)" (a model evolved from the "EndoPSI") in laparoscopy-assisted esophagojejunostomy together with the use of a circular stapler during laparoscopy-assisted total gastrectomy (LATG).

\section{Patients and methods}

\section{Patients}

This study included 23 patients ( 18 men and 5 women) and was done during the period May 2007 to May2008. All the patients were diagnosed preoperatively with gastric cancer (T1N0M0 stage IA or T2N0M0 stage IB, or T2N1M0 stage II, according to the International Union Against Cancer (UICC) classification of gastric cancer [4]). After obtaining adequate informed consent from the patients, LATG was performed.

\section{Methods}

Materials. The new PSI Endo-PSI (II) was developed in cooperation with Hope Electronics (Chiba, Japan; Fig. 1A). This device is a model evolved from the Endo-PSI. 


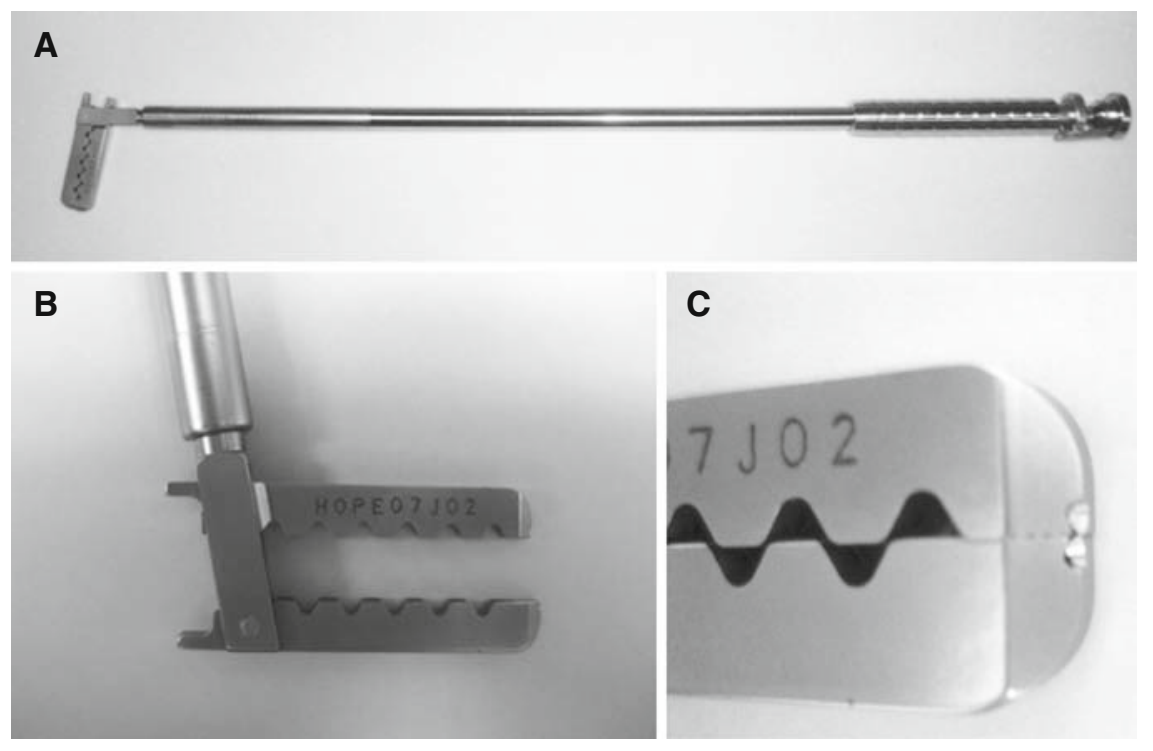

Fig. 1. A The newly developed pursestring suture instrument "Endo-PSI (II)". B The two jaws move in a parallel fashion when this device is opened or closed. The protruding part of the needle is covered by the projection of the jaw when the needle is completely inserted into the device. $\mathbf{C}$ The entrance hole of the device is cut in the shape of a slant line, and it is easy to check it laparoscopically
This device consists of two separate parts - jaws and a shaft with a grip — and it can be attached to a 12-mm trocar. The two jaws move in a parallel fashion when this device is opened or closed (Fig. 1B). So it can be attached to the abdominal esophagus easily in a laparoscopic view.

The width of the jaws is $50 \mathrm{~mm}$, which is fairly small in comparison with the conventional PSI used for open surgery. 2-0 Polypropylene suture (Surgipro; U.S. Surgical, USA) straight needle, $51 \mathrm{~mm}$ in length, is fitted to the Endo-PSI (II). When the needle is completely inserted into the device, the tip of the needle protrudes from the other side of the hole in the jaw. This protruding part of the needle is covered by the projection of the jaw, preventing damage from it to the organs around the esophagus, such as the liver. This mechanism supersedes that of the Endo-PSI. The entrance hole of the device is cut in the shape of a slant line (Fig. 1C). Thus, it is easy to check the entrance hole laparoscopically, and insertion of the straight needle can be performed easily. The safety of this device was checked in animal experiments on a pig before clinical application was conducted.

LATG operative technique. We performed reconstruction during LATG by a Roux-en-Y method or a Rouxen-Y with jejunal pouch method.

After the induction of general anesthesia, the patient was placed in a reverse Trendelenburg position, with the legs apart. After pneumoperitoneum was established, five ports were placed, i.e., bilateral subcostal, bilateral mid-abdominal, and infraumbilical ports $(12 \mathrm{~mm}$ each). The greater curvature and lesser curvature were mobilized adequately to expose the esophageal hiatus and abdominal esophagus. Radical lymphadenectomy for gastric cancer was performed in all patients. In the process of mobilization, duodenal transection was performed laparoscopically with an endoscopic linear stapler (ETS45; Ethicon EndoSurgery, Cincinnati, OH, USA). The duodenal stump was oversewn manually.

A 4-cm vertical median incision was made at the epigastrium and a Lap Disc (Hakko Shoji, Tokyo, Japan) was attached to the minilaparotomy. The EndoPSI (II) with a 12-mm trocar was inserted into the abdominal cavity, the Lap Disc was closed, and pneumoperitoneum was established. The Endo-PSI (II) was attached to the abdominal esophagus, and a straight needle with 2-0 polypropylene suture (Surgipro; U.S. Surgical) was passed through the Endo-PSI (II) (Fig. 2A, B) laparoscopically. Next, a surgical knot was made in advance and the thread was cut about $6 \mathrm{~cm}$ on the distal side of the knot after clipping. The abdominal esophagus was transected laparoscopically. The removed stomach was pulled out through the minilaparotomy, the anvil head of the circular stapler was placed in the abdominal cavity, and the Lap Disc was closed. The anvil head was inserted into the esophagus using an endoscopic anvil grasper and the purse-string suture was closed laparoscopically. The clip was able to be removed easily, even if the threads were pulled at the opposite side (Fig. 2C). And at the same time, the first ligation was also able to be done easily (Fig. 2D). After several ligations had been done, an Endo-loop (Ethicon Endo-Surgery) was also added to the proximal side of the first suture.

Reconstruction was performed by the Roux-enY method or by a Roux-en-Y with jejunal pouch method.

The jejuno-jejunal anastomosis was performed extracorporeally, and the distal limb of the jejunum was brought up anteriorly to the colon. After a purse-string 


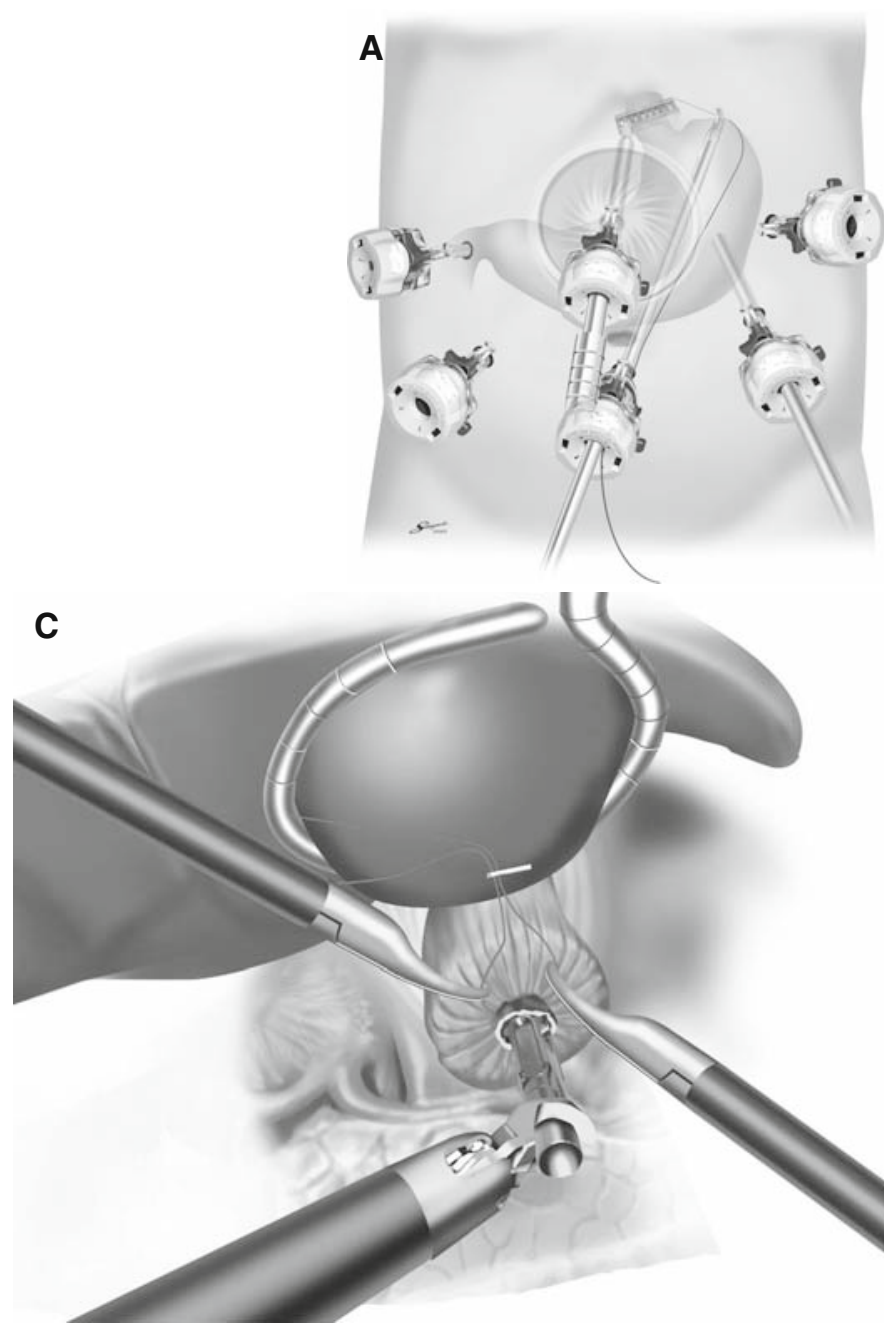

Fig. 2. A The Endo-PSI (II) with $12-\mathrm{mm}$ trocar was attached to a Lap Disc (Hakko Shoji), as shown. The needle holder for laparoscopic surgery was inserted into the infraumbilical trocar and the laparoscope was inserted into the left midabdominal trocar. B It is easy to check the entrance hole lapa-

suture was made at the stump of the distal limb of the jejunum, using the Endo-PSI (II), the body of the circular stapler was introduced into the distal limb of the jejunum (Fig. 3A). The Lap Disk was closed and pneumoperitoneum was established. The circular stapler was combined with the anvil head, laparoscopically (Fig. 3B, C). In this process, by pulling up the 2-0 polypropylene purse-string suture of the jejunum moderately, the slack of the jejunum anastomosis could be taken up.

The jejunal stump was closed laparoscopically with a 45-mm endoscopic linear stapler (ETS45; Ethicon Endo Surgery). In this process, lifting up the 2-0 polyproprolene suture intracorporeally, the endoscopic linear stapler could be easily attached to the appropriate point (Fig. 3D).
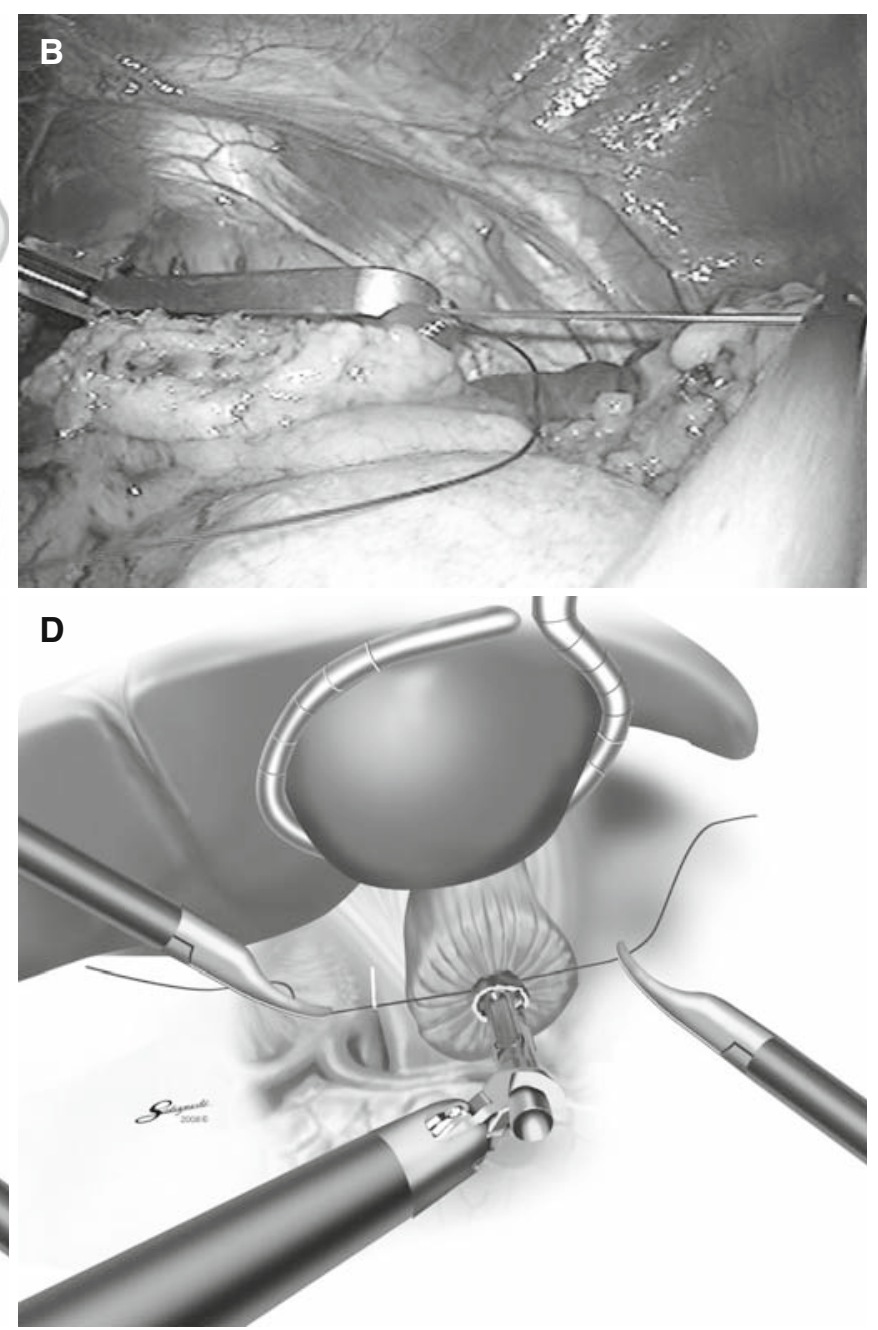

roscopically, and insertion of the straight needle can be performed easily. C The clip could be removed easily, even if the threads were pulled at the opposite side. D And at the same time, the first ligation could also be done easily

The abdominal cavity was checked, a silicon drainage tube was placed through the trocar wounds around the esophagojejunal anastomosis, and the incision was closed.

\section{Results}

All the patients underwent LATG and laparoscopic esophagojejunostomy without any intraoperative complications. No case required conversion to an open procedure. The characteristics of the patients are shown in Table 1.

A total of 15 patients underwent the Roux-en-Y method and 8 patients underwent the Roux-en-Y with jejunal pouch method. The body mass index (BMI) was 

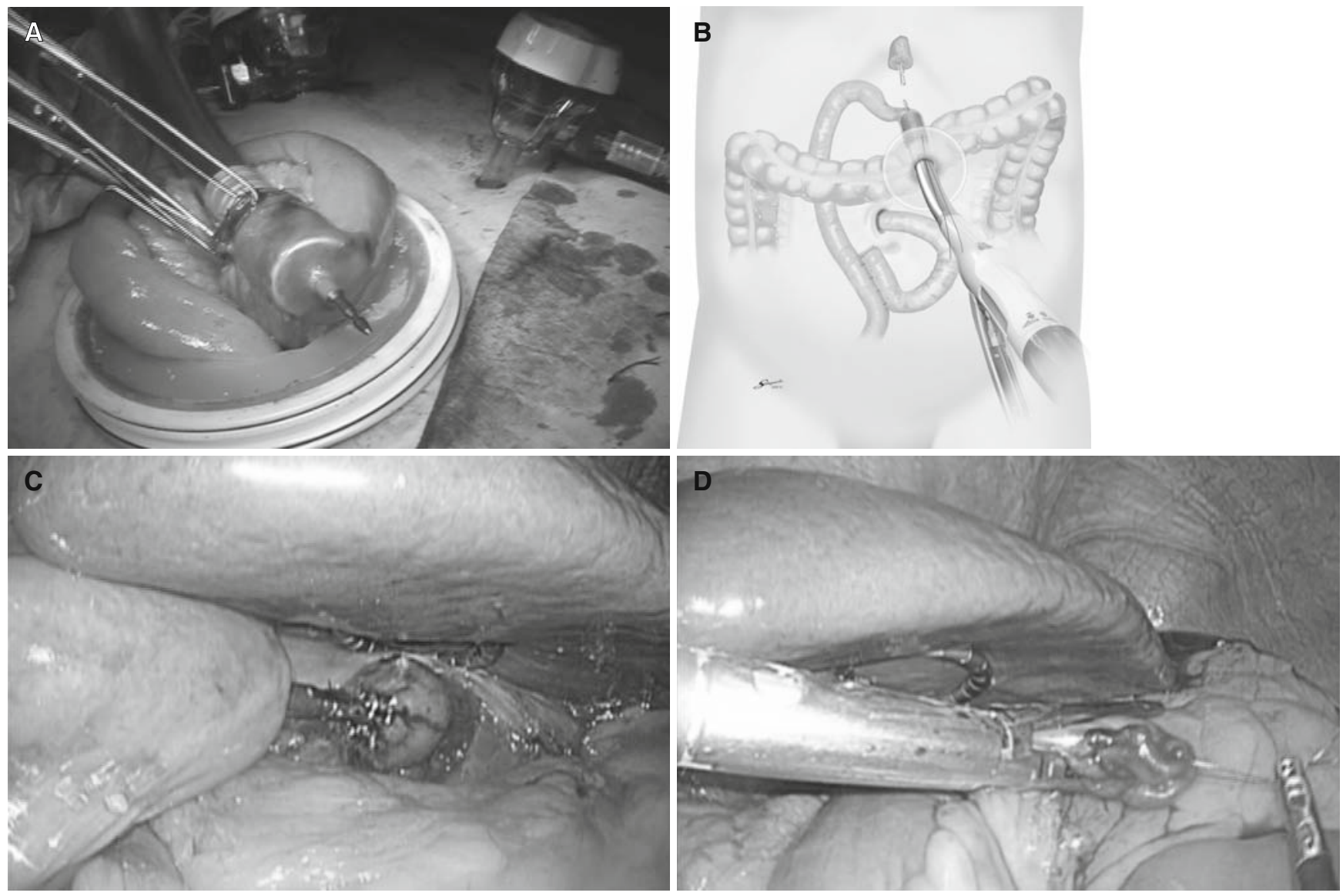

Fig. 3. A After the purse-string suture was made at the stump of the distal limb of the jejunum, using the Endo-PSI (II), the bination of the circular stapler with the anvil head was performed laparoscopically. D Lifting up the 2-0 polypropylene body of the circular stapler was introduced into the distal limb of the jejunum. B The circular stapler was placed in the abdominal cavity and the Lap Disk was closed. C The comsuture intracorporeally, the jejunal stump was closed with a 45-mm endoscopic linear stapler

Table 1. Characteristics of the patients

\begin{tabular}{lccc}
\hline Factor & $\begin{array}{c}\text { LATG (R-Y) group } \\
(n=15)\end{array}$ & $\begin{array}{c}\text { LATG (P-R-Y) group } \\
(n=8)\end{array}$ & $\begin{array}{c}\text { Total } \\
(n=23)\end{array}$ \\
\hline Male:Female & $12: 3$ & $6: 2$ & $18: 5$ \\
Age (years) & $67.9 \pm 12.8$ & $67.5 \pm 8.8$ & $67.7 \pm 11.5$ \\
BMI & $24.5 \pm 3.7$ & $22.3 \pm 2.5$ & $23.7 \pm 3.5$ \\
\hline
\end{tabular}

Table 2. Patients' operative details and postoperative hospital stay

\begin{tabular}{lccc}
\hline Factor & LATG (R-Y) group & LATG (P-R-Y) group & Total \\
\hline Operation time (min) & $301 \pm 58.3$ & $314.5 \pm 59.0$ & $305.9 \pm 57.6$ \\
Lymphadenectomy (D1 + B/D2) & $15 / 0$ & $7 / 1$ & $23 / 1$ \\
Blood loss (ml) & $83.6 \pm 70.3$ & $65.0 \pm 54.9$ & $77.5 \pm 71.7$ \\
Postoperative hospital stay (days) & $11.7 \pm 6.3$ & $10.0 \pm 0.89$ & $11.2 \pm 5.3$ \\
\hline
\end{tabular}

slightly higher in the Roux-en-Y group (R-Y group) than in the Roux-en-Y with jejunal pouch group (P-R-Y group).

The patients' operative details are summarized in Table 2. For the entire cohort, the mean operating time and the mean blood loss were $305.9 \pm 57.6 \mathrm{~min}$ and $77.5 \pm 71.7 \mathrm{ml}$ (mean $\pm \mathrm{SD}$ ), respectively, and the mean postoperative hospital stay was $11.2 \pm 5.3$ days. Roux stasis syndrome occurred in one patient in the R-Y group and this patient needed a postoperative hospital 
stay of 33 days. There were no other postoperative complications and there was no mortality.

\section{Discussion}

In laparoscopic surgery, we are aiming to reproduce the results of open surgery. So, a circular stapler was employed for the esophagoenteral anastomosis, instead of a side-to-side anastomosis using an endoscopic linear stapler.

We have already reported the use of a circular stapler and the Endo-PSI for an endoscopic esophagojejunal anastomosis, in 2007 [3]. The Endo-PSI was developed for hand-assisted surgery, because radical lymphadenectomy for LATG was performed using a HALS techniques before 2003 [5]. But, recently radical lymphadenectomy has been performed without HALS. So, a PSI that could be used without HALS techniques was needed.

There are two ways of using the Endo-PSI (II). One is that it is inserted directly into the minilaparotomy, as has been described for the first time in this report. The other is that the shaft is inserted into a $12-\mathrm{mm}$ trocar that has already been placed in the patient's body, and the jaw is attached to the tip of the shaft, which is pulled out from the abdominal cavity through the minilaparotomy. For inserting the anvil head of a circular stapler into the abdominal cavity, a minilaparotomy of at least $3-\mathrm{cm}$ is needed. These methods of using the Endo-PSI (II) are thought to be reasonable.

There are several reports about esophagoenteral anastomoses using circular staplers. Parker et al. [6] reported a double-stapling technique for esophagojejunostomy, in which the anvil head was placed in the flanged end of an oropharyngeal gastric tube. Hiki et al. [7] reported a modified double-stapling technique for esophagogastrostomy using a circular stapler. These are novel methods, which are not in need of conventional anvil insertion into the esophagus. Meanwhile, our method of anvil insertion is similar to the conventional method in open surgery. We think that laparoscopic anvil insertion will not be difficult, if we become accustomed to it. The entry hole of the esophagus is visible laparoscopically, rather than that in the direct view in open surgery.

The closure of the purse-string suture is able to be done easily, regardless of whether extracorporeal ligation or intracorporeal ligation is done. Also, the additional closure using the Endo-loop will be effective to fix the anvil head.
Recently, in LATG procedures at our institution, the Roux-en-Y method has been used more frequently than the Roux-en-Y with jejunal pouch method. The Rouxen-Y with jejunal pouch method is better than the Roux-en-Y method in terms of the amount of food that can be ingested, especially early after the operation. But the Roux-en-Y with jejunal pouch method is complicated and it was not easy to reproduce it laparoscopically, especially in obese patients. In obese patients, we have to choose the route of reconstruction carefully. If it is hard to perform the esophagojejunal anastomosis anteriorly to the colon, the route must be changed so that the anastomosis is performed posteriorly to the colon.

We always make sure, laparoscopically, that the distal limb of the jejunum can reach the esophagus, before the stapler is fired.

The optimal method of reconstruction in LATG still remains to be established. But we believe that laparoscopic esophagojejunostomy using a circular stapler and the Endo-PSI (II) is suitable and feasible. The technique may also be applied to esophagogastrostomy performed during laparoscopic proximal gastrectomy (LAPG).

Acknowledgments We thank Shigeyuki Sakaguchi for the preparation of the schema.

\section{References}

1. Uyama I, Sugioka A, Fujita J, Komori Y, Matsui H, Hasumi A. Laparoscopic total gastrectomy with distal pancreatosplenectomy and D2 lymphadenectomy for advanced gastric cancer. Gastric Cancer 1999;2:230-4.

2. Matsui H, Uyama I, Sugioka A, Fujita J, Komori Y, Ochiai M, et al. Linear stapling forms improved anastomoses during esophagojejunostomy after a total gastrectomy. Am J Surg 2002;184:58-60.

3. Usui S, Ito K, Hiranuma S, Takiguchi N, Matsumoto A, Iwai T. Hand-Assisted laparoscopic esophagojejunostomy using newly developed purse-string suture instrument "Endo-PSI". Surg Laparosc Endosc Percutan Tech 2007;17:107-10.

4. Sobin LH, Wittekind C. International Union Against Cancer: TNM classification of malignant tumors. 5 th ed. New York: WileyLiss; 1997:59-62.

5. Usui S, Inoue H, Yoshida T, Fukami N, Kudo SE, Iwai T. Handassisted laparoscopic total gastrectomy for early gastric cancer. Surg Laparosc Endosc Percutan Tech 2003;13:304-7.

6. Parker J, Sell H, Stahlfeld K. A new technique for esophagojejunostomy after total gastrectomy for gastric cancer. Am J Ssurg 2001;182:174-6.

7. Hiki N, Fukunaga T, Yamaguchi T, Nunobe S, Tokunoga M, Ohyama $\mathrm{S}$, et al. Laparoscopic esophagogastric circular stapled anastomosis: a modified technique to protect the esophagus. Gastric Cancer 2007;10:181-6. 